\title{
Temporal relationship of computed and structured diagnoses in electronic health record data
}

\author{
Wade L. Schulz ${ }^{1,2}$, H. Patrick Young 2,3 , Andreas Coppi ${ }^{2,3}$, Bobak J. Mortazavi 2,3,4,5, Zhenqiu Lin², \\ Raymond A. Jean ${ }^{2,6}$ and Harlan M. Krumholz ${ }^{2,3,7^{*}}$ (1)
}

\begin{abstract}
Background: The electronic health record (EHR) holds the prospect of providing more complete and timely access to clinical information for biomedical research, quality assessments, and quality improvement compared to other data sources, such as administrative claims. In this study, we sought to assess the completeness and timeliness of structured diagnoses in the EHR compared to computed diagnoses for hypertension (HTN), hyperlipidemia (HLD), and diabetes mellitus (DM).

Methods: We determined the amount of time for a structured diagnosis to be recorded in the EHR from when an equivalent diagnosis could be computed from other structured data elements, such as vital signs and laboratory results. We used EHR data for encounters from January 1, 2012 through February 10, 2019 from an academic health system. Diagnoses for HTN, HLD, and DM were computed for patients with at least two observations above threshold separated by at least 30 days, where the thresholds were outpatient blood pressure of $\geq 140 / 90 \mathrm{mmHg}$, any lowdensity lipoprotein $\geq 130 \mathrm{mg} / \mathrm{dl}$, or any hemoglobin $\mathrm{A} 1 \mathrm{c} \geq 6.5 \%$, respectively. The primary measure was the length of time between the computed diagnosis and the time at which a structured diagnosis could be identified within the EHR history or problem list.
\end{abstract}

Results: We found that $39.8 \%$ of those with HTN, 21.6\% with HLD, and 5.2\% with DM did not receive a corresponding structured diagnosis recorded in the EHR. For those who received a structured diagnosis, a mean of 389, 198, and 166 days elapsed before the patient had the corresponding diagnosis of HTN, HLD, or DM, respectively, recorded in the EHR.

Conclusions: We found a marked temporal delay between when a diagnosis can be computed or inferred and when an equivalent structured diagnosis is recorded within the EHR. These findings demonstrate the continued need for additional study of the EHR to avoid bias when using observational data and reinforce the need for computational approaches to identify clinical phenotypes.

Keywords: Real-world data, Electronic health records, Observational studies, Computational phenotypes

*Correspondence: harlan.krumholz@yale.edu

${ }^{2}$ Center for Outcomes Research and Evaluation, Yale New Haven Hospital,

New Haven, CT, USA

Full list of author information is available at the end of the article

\section{Background}

Despite the rapid digitization of healthcare, the current research enterprise remains inefficient. Randomized control trials (RCTs), which remain the gold standard, are costly, time-consuming, and capture only a small crosssection of patients, which limits their generalizability [1, 
2]. To accelerate the pace of discovery, investigators and regulatory agencies have increasingly focused on realworld data (RWD), defined as data collected outside of a traditional research environment, as a source of information [3-5]. Real-world data sources include administrative claims and discharge databases, clinical registries, and electronic health records (EHRs), among others. Despite increased access to these digital data, it remains important for investigators to be aware of and account for limitations in these observational data sets during study design and analysis [6-9].

The creation of patient cohorts and study endpoints often requires the identification of clinical diagnoses. These populations and endpoints are frequently characterized by diagnostic codes, such as International Statistical Classification of Diseases and Related Health Problems $10^{\text {th }}$ Revision Clinical Modification (ICD-10-CM) codes [10]. Observational and outcomes research have long used administrative claims and registries as a source of this information [11-18]. However, these repositories come with the known limitations of significant time delays in availability and a lack of detailed clinical records $[19,20]$. In addition, significant costs are associated with manual abstraction for diseasespecific registries [21]. Because of these limitations and the increased access to detailed EHR data, investigators have increasingly focused on the EHR to provide the data needed to support a wide range of studies.

Information obtained from the EHR has the potential to provide near real-time access to a more complete data set than can be provided from other RWD sources $[19,20]$. The EHR is the primary repository of a patient's clinical history, but the data elements needed to represent a patient's history can be found in many locations, from structured fields in the history and problem list to unstructured clinical notes [9]. Prior work has shown that patient history and problem lists within the EHR can be incomplete and contain frequent errors [22-25]. Even for a relatively straightforward diagnosis such as hypertension, researchers from the OneFlorida clinical data research network found that as many as $30 \%$ of those they identified with hypertension by means of clinical measurements recorded in the EHR were missing the associated structured diagnostic code [26], similar to results found by an earlier study from Stanford [27].

Yet studies based on EHR data frequently use, sometimes solely, structured diagnostic codes to create cohorts and identify outcomes [28, 29]. Since administrative claims are ultimately derived from these structured fields, the EHR is likely not significantly worse than claims-based sources. Investigators have also demonstrated that structured diagnostic codes can provide valuable information when analyzed in the appropriate context [30,31]. Therefore, while limitations to the use of structured diagnoses from the EHR exist, they remain frequently used and ongoing study can increase the value of results generated from these sources.

A primary goal of EHR-based studies is access to near real-time information [32,33], but it remains an indirect assessment of a patient's status due to how the EHR is used in clinical workflows [34]. While data may be extracted immediately, clinical workflows and decision making may impact the timeliness of data entry into the EHR, particularly within structured data elements. Hence, the extent to which structured diagnoses may be missing at the time of analysis may be greatly underestimated. Therefore, computed diagnoses may be needed in order provide more accurate and timely information. While no single approach to creating a computed phenotype is necessarily best, understanding the limitations of EHR and other RWD sources is crucial for appropriate study design and interpretation of results.

In this study, we determine how structured diagnostic codes in the patient history and problem list compare to computed diagnoses for hypertension (HTN), hyperlipidemia (HLD), and diabetes mellitus (DM). We selected these three diagnoses with because they can be efficiently computed from structured clinical and laboratory data. We extend on prior work in the field to identify not only the completeness of structured diagnostic codes, but also the temporal association between the computed diagnosis and manual recording of an equivalent diagnostic code within the clinical record.

\section{Methods \\ Data sources}

We created our data set from a complete extract of the Yale New Haven Health clinical data warehouse (Epic Caboodle) that was transformed into the PCORnet Common Data Model v3.1 (CDM) on February 11, 2019 using our local data analytics platform [35]. The Caboodle source tables and supporting terminology tables were transformed into the demographic, encounter, diagnosis, condition, lab_result_cm, and vital PCORnet CDM tables, which were used for analysis. Diagnosis and condition source were categorized as defined in the PCORnet CDM [36] for $O D, B I$, and $C L$ which we label as provider, billing, and claims. As a data quality study based on existing and deidentified data, this work was not classified as human subjects research and did not require Institutional Review Board approval.

\section{Phenotype definitions}

We collected information to assess HTN, HLD, and DM. We excluded data with dates earlier than January 1, 2012 as these represented a sparse fraction of 
the raw data with unreliable dates of onset due to their transfer between prior EHR systems. The most recent date of measurement or sample collection included in the analysis was restricted to on or before August 11, 2018, whereas diagnosis events were current up to February 10, 2019, allowing a period of at least 26 weeks (approximately six months) between the most recent measurement or result and the final date available. If multiple measurements were performed on the same day, then the minimum value of the measurement was selected to provide the most restrictive threshold and, consequently, a conservative assessment of the possible disease condition. Only outpatient blood pressure readings, as annotated within the PCORnet CDM, were used, but laboratory results were extracted from all encounter settings. Relevant laboratory results for LDL and A1c were identified by LOINC codes (LDL: '134577', '18262-6'; A1c: '4548-4') code or internal EHR codes (various and specific to our institution).

We flagged measurements as a 'signal of disease' whenever they exceed a specific threshold. For HTN, a measurement was flagged as a 'signal of disease' if either the minimum systolic reading was $\geq 140 \mathrm{mmHg}$ or the minimum diastolic reading was $\geq 90 \mathrm{mmHg}$. For HLD, the threshold was an LDL $\geq 130 \mathrm{mg} / \mathrm{dL}$ and for $\mathrm{DM}$ the threshold was an $\mathrm{A} 1 \mathrm{c} \geq 6.5 \%$. For all three conditions, if any two measurements taken at least 30 days apart were found above the threshold (abnormal is high in all three cases), the patient was considered to have a computed diagnosis of disease on the date of the second signal.
For each condition and diagnosis code system, the first 3 or 5 characters of the code string were matched against ICD-10-CM and ICD-9-CM parent codes (Table 1). Patients with a diagnosis present prior to the first signal were flagged as having an existing diagnosis. For patients without a prior diagnosis or computed diagnosis as defined above, the first date a matching diagnosis code (Table 1) was recorded in the EHR, if present, was logged along with its origin (provider-entered billing diagnosis; provider-entered encounter diagnosis or problem list entry; or diagnosis code from returned claims) and the date of the patient's most recent encounter with the health system. A final data set consisting of the computed signal dates, computed and structured diagnosis dates, date of most recent encounter, and diagnosis origin was used for analysis.

\section{Data analysis and statistical approaches}

Data extraction was done with custom PySpark scripts using Spark (v2.1.0). Preprocessing and summary statistics were performed using the pandas (v0.24.1) and NumPy (v1.16.2) Python libraries. Visualizations were produced with the Matplotlib (v3.0.3) and seaborn (v0.9.0) Python libraries. To model the time to diagnosis, we employed the Kaplan-Meier estimation method for survival analysis using the lifelines (v0.20.0) Python library. All study-specific scripts were reviewed by an independent analyst.

Patients with an existing (prior to first signal) or early (recorded between the first and second signal) diagnosis

Table 1 Diagnosis criteria and ICD-9-CM and ICD-10-CM codes used to determine computed and recorded diagnoses

\begin{tabular}{|c|c|}
\hline Condition & Signal and diagnosis criteria \\
\hline \multirow[t]{5}{*}{ Hypertension } & Denominator: Outpatient systolic $\geq 140$ or diastolic $\geq 90(\mathrm{mmHg})$ \\
\hline & 2 readings $\geq 30$ days apart and no prior diagnosis \\
\hline & Numerator: New diagnosis of any of the following \\
\hline & ICD-9 groups: 401, 402, 403, 404, 405, 642 \\
\hline & ICD-10 groups: $|10| 11,,|12| 13,,|15| 16,, \mid 27,013$ \\
\hline \multirow[t]{5}{*}{ Diabetes } & Denominator: $\mathrm{A} 1 \mathrm{c} \geq 6.5(\%)$ \\
\hline & 2 readings $\geq 30$ days apart and no prior diagnosis \\
\hline & Numerator: New diagnosis of any of the following \\
\hline & ICD-9 groups: 249,250 \\
\hline & ICD-10 groups: E08, E09, E10, E11, E13 \\
\hline \multirow[t]{5}{*}{ Hyperlipidemia } & Denominator: LDL $\geq 130$ (mg/dL) \\
\hline & 2 readings $\geq 30$ days apart and no prior diagnosis \\
\hline & Numerator: New diagnosis of any of the following \\
\hline & ICD-9: 272.0, 272.2, 272.3, 272.4, 272.9 \\
\hline & ICD-10: E78.0, E78.2, E78.3, E78.4, E78.5, E78.7, E78.8, E78.9 \\
\hline
\end{tabular}


were excluded from the time to diagnosis analysis. The duration for the survival analysis (equivalent to "survival time") was the number of days between the date of the second signal and the date of diagnosis. For those who were never diagnosed, it was defined as the number of days between the date of the second signal and the date of the most recent encounter, at which point they were censored due to lack of additional follow-up.

\section{Results}

\section{Frequency of clinical and computed diagnoses}

We defined a signal of disease as an observation above the threshold. In our cohort, we computed a diagnosis of HTN in 245,711 patients, HLD in 45,098 patients, and DM in 45,460 patients who met our criteria for two signals separated by at least thirty days. Of these patients, a pre-existing, structured diagnosis of HTN, HLD, or DM, was present in the EHR for $42.0 \%, 37.4 \%$, and $54.8 \%$ of patients, respectively, before the first signal was identified (Fig. 1). For patients with a new diagnosis, there was a large degree of variability in the presence of structured diagnostic codes among the conditions we assessed. For DM, $76.5 \%$ of patients received an early structured diagnosis, meaning a structured diagnosis was recorded in the EHR in the window between the first and second signal. However, for those with HTN or HLD, only $36.4 \%$ and $51.3 \%$, respectively, received an early clinical diagnosis. Similarly, $39.8 \%$ of those with computed HTN and 21.6\% of those with a computed HLD diagnosis never received a structured diagnosis in the EHR, while only $5.2 \%$ of those with DM lacked a structured, clinical diagnosis.

\section{Origin of first clinical diagnosis}

We also assessed the source of the first structured diagnosis to determine whether it came from a providerentered, billing, or claims-based diagnosis. For structured diagnoses of different sources that were recorded on the same date, the provider-entered entry took priority, with billing second and claims third in order of precedence. We found that the first source was similar among all three conditions, with most diagnoses being providerentered within the medical history or problem list, followed by billing-related diagnoses (Fig. 2). Only a small proportion of diagnoses were first identified via returned claims within our local data set.

\section{Temporality of clinical and computed diagnoses}

Since the timing of diagnosis is relevant to cohort creation, we determined the delay in availability of the first structured diagnosis compared with when a diagnosis could be computed from other data available in the EHR. To define a consistent starting time, patients with a pre-existing diagnosis (present prior to first signal) or early diagnosis (occurring between the first and second signal) were excluded. Within this cohort, the mean time for a structured diagnosis to be recorded ranged from a minimum of 166 days for DM to a maximum of nearly 600 days for HTN (Fig. 3a) from the time of the computed diagnosis. The temporal delay varied by the source of diagnosis, with provider-entered diagnoses having the

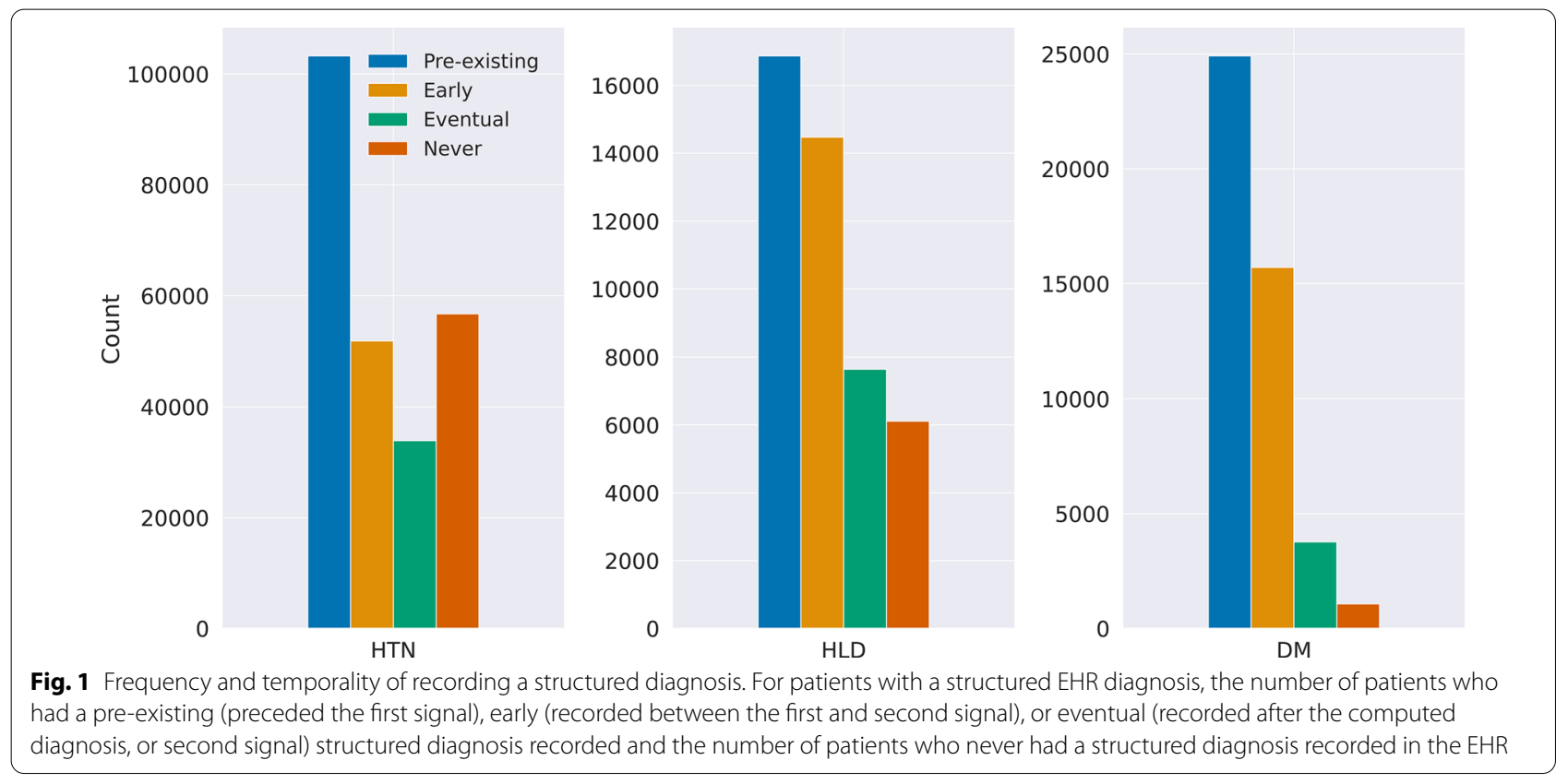




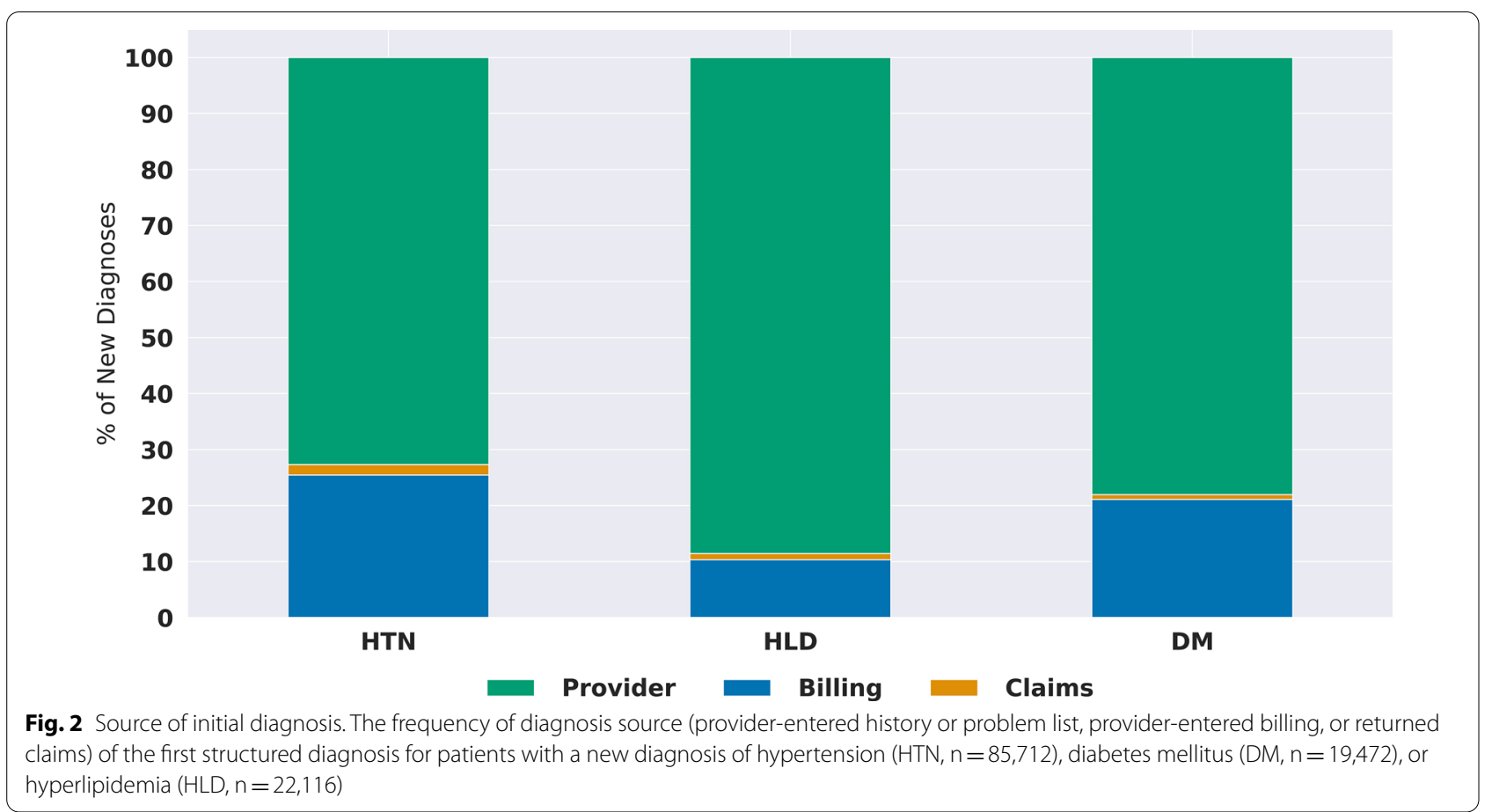

shortest interval in all conditions and claims-based diagnoses having the longest. It should be noted that very few patients had a claim as the first occurrence of a structured diagnosis $(n=633,128$, and 33 for HTN, HLD, and $\mathrm{DM}$, respectively).

To assess the timeliness of structured diagnoses, we estimated the likelihood of having a structured diagnosis present in the EHR using the Kaplan-Meier estimation method, where the last encounter was used as the censor date if a discrete diagnosis was not found. The likelihood of not having a structured diagnosis also varied by condition (Fig. 3b). While those with DM had nearly an $80 \%$ chance of having a structured diagnosis recorded at 2 years, those with HLD had less than a 60\% chance and those with HTN had less than a $40 \%$ chance of having a structured diagnosis present at the same timepoint. The mean time from the second signal of disease to censoring for those who did not receive a diagnosis was 726 days, 600 days, and 575 days for HTN, HLD, and DM, respectively.

\section{Discussion}

The primary finding of this study was that structured diagnoses within the EHR for HTN, HLD, and DM can have a marked delay in being recorded compared with the time a diagnosis can be computed from other EHR data. For three common diseases, the average time from which a diagnosis could be computed from laboratory values preceded the manual recording of a structured diagnosis by as much as 389 days. In addition, even one year after a diagnosis can be computed, a large percentage of patients do not have an equivalent structured diagnosis recorded in the EHR. Therefore, while the EHR has several potential advantages to other sources of RWD and can be accessed in near real-time from a technical perspective, the recording of clinical information within the structured history and problem list may be less sensitive and delayed compared to identifying computed diagnoses for certain conditions. Thus, studies based on RWD, the approach to extracting information from the EHR may affect its quality.

The EHR contains a detailed record of a patient's clinical history but extracting this history from the structured and unstructured fields that data can reside in remains a challenge. Our work extends the prior literature, which have focused on the completeness and accuracy of the problem list compared to manual adjudication or next generation phenotyping approaches $[22,23,25,30,37,38]$. The delay in recording a structured diagnosis has the potential to impact the development of cohorts and outcome ascertainment from RWD because analyses based on structured diagnostic codes could preferentially identify patients with a longer history of disease. In addition, analyses limited to patients with more recent data are likely to have a lower prevalence of disease than cohorts with a longer history, which may bias historic comparisons with synthetic or external control arms. Finally, if data are 

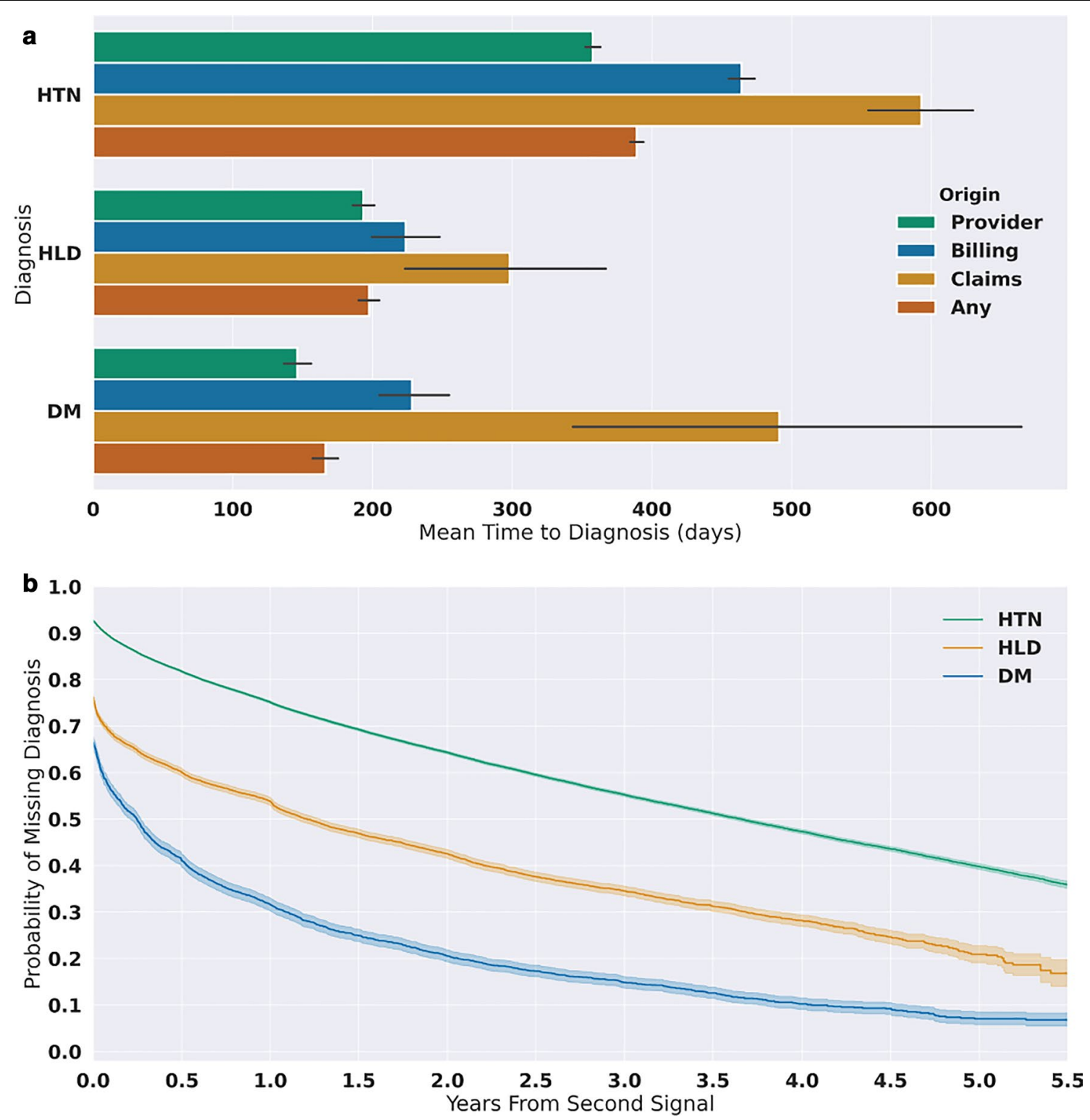

Fig. 3 Temporal delay between computed and recorded diagnoses. a The mean time in days to receive a structured diagnosis from the time of the second signal. Means were calculated using the number of days to initial diagnosis. $\mathbf{b}$ Kaplan-Meier estimated probability that a patient is missing a structured diagnosis after a computed diagnosis is made (presence of two signals) for patients with hypertension (HTN, $n=90,614)$, diabetes mellitus ( $D M, n=4836)$, or hyperlipidemia (HLD, $n=13,754)$

obtained from multiple institutions with varying local diagnostic patterns, additional biases may be introduced to multi-site studies.

While concerns of EHR data completeness are often described as data collection and quality issues, this is, in many cases, primarily a concern when the data are used for secondary research purposes [6, 39, 40]. When assessed from a clinical perspective, information related to a disorder, such as blood pressure measurements or documentation within an unstructured clinical note, can be used by a healthcare provider to draw equivalent conclusions, despite the high potential to be missed during automated digital extraction. Therefore, EHR data may not be missing or of low quality, but are rather collected for clinical, rather than research, purposes. Even with these limitations, EHR data can add significant value when analyzed appropriately. For example, others have demonstrated that what may often be described as noise within EHR data, such 
as frequency of measurements or presence of repeat diagnoses, can actually be used to predict patient outcome and the temporality of clinical conditions [31, 34, 41].

Despite the potential concerns related to the use of EHR described here, it is important to acknowledge that similar issues can also be found in clinical research and manually adjudicated data sets, such as disease registries. Several studies have shown significant variability in the accuracy and inter-rater reliability of manual data abstraction. One case study by the Office of the Inspector General for the Department of Health and Human Services found that manual nurse review identified $78 \%$ (93 of 120) of adverse events in the study population [42]. Similarly, patient report, a common source for clinical research studies, has been found to over- or under-represent even major healthcare events, such as readmission, in nearly $30 \%$ of cases [43]. Therefore, strategies to better understand and use RWD to augment data collected through traditional methods have the potential to increase the accuracy and completeness of patient history, clinical events, and healthcare outcomes.

This study has several limitations. First, data were collected from a single site within a healthcare system. We did not assess the possible impact of data quality issues in the EHR and its mapping to the PCORnet Common Data Model, a complex issue we consider to be beyond the scope of this study. However, our findings for the number of missing diagnoses for those with HTN were consistent with previously published studies $[26,27]$. This work also focused on only three phenotypes which could be reliably identified from clinical measurements and laboratory testing, all of which were chronic diseases, and did not assess for more complex diagnoses or variations in thresholds for diagnosis. Finally, we did not assess the cause or clinical impact of delayed or missing structured diagnoses.

While strategies to assess data quality and account for variations in data collection for clinical research data have been developed, access to and use of RWD remains a new and rapidly evolving field. Like diagnostic laboratory tests, methods to extract data from the EHR can be viewed as assays with varying sensitivity, specificity, and window periods. Work by the Electronic Medical Records and Genomics (eMERGE) [44] and OHDSI [38, 45] networks, among others, to create standardized next generation phenotypes will continue to improve our ability to identify clinical cohorts and outcomes. While no single approach may be effective for every study, standardized strategies to assess whether RWD and specific computed phenotypes are fit-for-purpose will need ongoing, and likely use case-specific, assessment.

\section{Conclusions}

We found that the recording of structured diagnoses within the EHR had a marked time delay compared to when a computed diagnosis could be extracted from clinical findings and laboratory results within the EHR. The delay and presence of a structured diagnosis varied by disease. These findings highlight the need for continued assessment of RWD analysis and the validation of EHR data when used for biomedical research.

\section{Abbreviations}

EHR: Electronic health record; HTN: Hypertension; HLD: Hyperlipidemia; DM: Diabetes mellitus; RCT: Randomized control trial; ICD-10-CM: International Statistical Classification of Diseases and Related Health Problems, 10th Revision Clinical Modification; CDM: Common data model.

\section{Acknowledgements}

We would like to acknowledge Aeron Small for his review of this manuscript. We would also like to acknowledge Charlie Torre, Jr, William Byron, and Nathaniel Price for their assistance with maintaining the underlying infrastructure for the data used in this analysis.

\section{Authors' contributions}

W.S. conceived the study and led the design and analysis. P.Y. carried out the data extraction and analyses. A.C. assisted with data analysis and independent code review. W.S., P.Y., A.C., B.M., Z.L., R.J., and H.K. discussed results and implications and all contributed to the writing and editing of the manuscript. All authors have approved the final manuscript.

\section{Funding}

Not applicable.

\section{Availability of data and materials}

The data sets analysed during the current study are considered sensitive and proprietary and hence are not publicly available. Further details are available from the corresponding author on reasonable request.

\section{Ethics approval and consent to participate}

As a data quality study based on existing and deidentified data, this work was not classified as human subjects research and did not require Institutional Review Board approval.

\section{Consent for publication}

Not applicable.

\section{Competing interests}

Harlan Krumholz works under contract with the Centers for Medicare \& Medicaid Services to support quality measurement programs; was a recipient of a research grant, through Yale, from Medtronic and the US Food and Drug Administration to develop methods for post-market surveillance of medical devices; was a recipient of a research grant with Medtronic and is the recipient of a research grant from Johnson \& Johnson, through Yale University, to support clinical trial data sharing; was a recipient of a research agreement, through Yale University, from the Shenzhen Center for Health Information for work to advance intelligent disease prevention and health promotion; collaborates with the National Center for Cardiovascular Diseases in Beijing; receives payment from the Arnold \& Porter Law Firm for work related to the Sanofi clopidogrel litigation, from the Ben C. Martin Law Firm for work related to the Cook Celect IVC filter litigation, and from the Siegfried and Jensen Law Firm for work related to Vioxx litigation; chairs a Cardiac Scientific Advisory Board for UnitedHealth; was a participant/participant representative of the IBM Watson Health Life Sciences Board; is a member of the Advisory Board for Element Science, the Advisory Board for Facebook, and the Physician Advisory Board for Aetna; and is the co-founder of HugoHealth, a personal health information platform, and co-founder of Refactor Health, an Al-augmented data management platform for healthcare. Wade Schulz was an investigator 
for a research agreement, through Yale University, from the Shenzhen Center for Health Information for work to advance intelligent disease prevention and health promotion; collaborates with the National Center for Cardiovascular Diseases in Beijing; is a technical consultant to HugoHealth, a personal health information platform, and co-founder of Refactor Health, an Al-augmented data management platform for healthcare; is a consultant for Interpace Diagnostics Group, a molecular diagnostics company. The other co-authors report no potential competing interests.

\section{Author details}

${ }^{1}$ Department of Laboratory Medicine, Yale School of Medicine, New Haven, CT, USA. ${ }^{2}$ Center for Outcomes Research and Evaluation, Yale New Haven Hospital, New Haven, CT, USA. ${ }^{3}$ Section of Cardiovascular Medicine, Department of Internal Medicine, Yale School of Medicine, New Haven, CT, USA. ${ }^{4}$ Department of Computer Science and Engineering, Texas A\&M University, College Station, TX, USA. ${ }^{5}$ Center for Remote Health Technologies and Systems, Texas A\&M University, College Station, TX, USA. ${ }^{6}$ Department of Surgery, Yale School of Medicine, New Haven, CT, USA.' Department of Health Policy and Management, Yale School of Public Health, New Haven, CT, USA

Received: 7 September 2020 Accepted: 31 January 2021

Published online: 17 February 2021

\section{References}

1. Mulder $\mathrm{R}$, Singh $A B$, Hamilton $A$, et al. The limitations of using randomised controlled trials as a basis for developing treatment guidelines. Evid Based Ment Health. 2018;21(1):4-6. https://doi.org/10.1136/eb-2017102701.

2. Booth CM, Tannock IF. Randomised controlled trials and populationbased observational research: partners in the evolution of medical evidence. Br J Cancer. 2014;110(3):551-5. https://doi.org/10.1038/ bjc.2013.725.

3. Sherman RE, Anderson SA, Dal Pan GJ, et al. Real-world evidence-What is it and what can it tell us? N Engl J Med. 2016;375(23):2293-7. https:// doi.org/10.1056/NEJMsb1609216.

4. Miksad RA, Abernethy AP. Harnessing the power of real-world evidence (RWE): a checklist to ensure regulatory-grade data quality. Clin Pharmacol Ther. 2018;103(2):202-5. https://doi.org/10.1002/cpt.946.

5. Khosla S, White R, Medina J, et al. Real world evidence (RWE) — a disruptive innovation or the quiet evolution of medical evidence generation? [version 2; peer review: 2 approved]. F1000Res. 2018;7:111. https://doi. org/10.12688/f1000research.13585.2.

6. Hersh WR, Weiner MG, Embi PJ, et al. Caveats for the use of operational electronic health record data in comparative effectiveness research. Med Care. 2013;51 (8 Suppl 3):S30-7. https://doi.org/10.1097/MLR.0b013e3182 9b1dbd.

7. Kim H-S, Kim JH. Proceed with caution when using real world data and real world evidence. J Korean Med Sci. 2019;34(4):e28. https://doi. org/10.3346/jkms.2019.34.e28.

8. Ryan PB, Madigan D, Stang PE, Overhage JM, Racoosin JA, Hartzema AG Empirical assessment of methods for risk identification in healthcare data: results from the experiments of the Observational Medical Outcomes Partnership. Stat Med. 2012;31(30):4401-15. https://doi.org/10.1002/ $\operatorname{sim} .5620$

9. Häyrinen K, Saranto K, Nykänen P. Definition, structure, content, use and impacts of electronic health records: a review of the research literature. Int J Med Inform. 2008;77(5):291-304. https://doi.org/10.1016/j.ijmed inf.2007.09.001.

10. ICD - ICD-10-CM - International Classification of Diseases, Tenth Revision, Clinical Modification. Available at: https://www.cdc.gov/nchs/icd/icd10 cm.htm. Accessed December 2, 2019.

11. Mitchell JB, BubolzT, Paul JE, et al. Using Medicare claims for outcomes research. Med. Care 1994;32(7 Suppl):JS38-51.

12. Blumenthal $\mathrm{S}$. The use of clinical registries in the united states: $\mathrm{A}$ landscape survey. EGEMS (Wash. DC) 2017;5(1):26. https://doi.org/10.5334/ egems.248.

13. Birnbaum HG, Cremieux PY, Greenberg PE, LeLorier J, Ostrander JA, Venditti L. Using healthcare claims data for outcomes research and pharmacoeconomic analyses. Pharmacoeconomics. 1999;16(1):1-8. https ://doi.org/10.2165/00019053-199916010-00001.

14. Hoque DME, Kumari V, Hoque M, Ruseckaite R, Romero L, Evans SM. Impact of clinical registries on quality of patient care and clinical outcomes: a systematic review. PLoS ONE. 2017;12(9):e0183667. https://doi. org/10.1371/journal.pone.0183667.

15. Mues KE, Liede A, Liu J, et al. Use of the Medicare database in epidemiologic and health services research: a valuable source of real-world evidence on the older and disabled populations in the US. Clin Epidemiol. 2017;9:267-77. https://doi.org/10.2147/CLEP.S105613.

16. Krumholz HM, Lin Z, Drye EE, et al. An administrative claims measure suitable for profiling hospital performance based on 30-day all-cause readmission rates among patients with acute myocardial infarction. Circ Cardiovasc Qual Outcomes. 2011;4(2):243-52. https://doi.org/10.1161/ CIRCOUTCOMES.110.957498.

17. Ferver $K$, Burton B, Jesilow P. The use of claims data in healthcare research. Open Public Health J. 2009;2(1):11-24. https://doi.org/10.2174/18749 44500902010011.

18. The PCORI Methodology Report |PCORI. Available at: https://www.pcori org/research-results/about-our-research/research-methodology/pcori -methodology-report. Accessed December 3, 2019.

19. Jollis JG, Ancukiewicz M, DeLong ER, Pryor DB, Muhlbaier LH, Mark DB. Discordance of databases designed for claims payment versus clinical information systems. Implications for outcomes research. Ann. Intern. Med. 1993;119(8):844-850. https://doi.org/10.7326/0003-4819-119-8-19931 0150-00011.

20. Hartzema AG, Racoosin JA, MaCurdy TE, Gibbs JM, Kelman JA. Utilizing Medicare claims data for real-time drug safety evaluations:is it feasible? Pharmacoepidemiol Drug Saf. 2011;20(7):684-8. https://doi.org/10.1002/ pds.2143.

21. Subramanian S, Tangka FKL, Beebe MC, Trebino D, Weir HK, Babcock F. The cost of cancer registry operations: Impact of volume on cost per case for core and enhanced registry activities. Eval Program Plann. 2016;55:1-8. https://doi.org/10.1016/j.evalprogplan.2015.11.005.

22. Wright A, McCoy AB, Hickman T-TT, et al. Problem list completeness in electronic health records: a multi-site study and assessment of success factors. Int. J. Med. Inform. 2015;84(10):784-790. https://doi.org/10.1016/j. ijmedinf.2015.06.011.

23. Singer A, Kroeker AL, Yakubovich S, Duarte R, Dufault B, Katz A. Data quality in electronic medical records in Manitoba: Do problem lists reflect chronic disease as defined by prescriptions? Can Fam Physician. 2017;63(5):382-9.

24. Holmes C, Brown M, Hilaire DS, Wright A. Healthcare provider attitudes towards the problem list in an electronic health record: a mixed-methods qualitative study. BMC Med Inform Decis Mak. 2012;12:127. https://doi. org/10.1186/1472-6947-12-127.

25. Szeto HC, Coleman RK, Gholami P, Hoffman BB, Goldstein MK. Accuracy of computerized outpatient diagnoses in a Veterans Affairs general medicine clinic. Am J Manag Care. 2002;8(1):37-43.

26. Smith SM, McAuliffe K, Hall JM, et al. Hypertension in Florida: data from the Oneflorida clinical data research network. Prev Chronic Dis. 2018;15:E27. https://doi.org/10.5888/pcd15.170332.

27. Banerjee D, Chung S, Wong EC, Wang EJ, Stafford RS, Palaniappan LP. Underdiagnosis of hypertension using electronic health records. Am J Hypertens. 2012;25(1):97-102. https://doi.org/10.1038/ajh.2011.179.

28. Hripcsak G, Ryan PB, Duke JD, et al. Characterizing treatment pathways at scale using the OHDSI network. Proc Natl Acad Sci USA. 2016;113(27):7329-36. https://doi.org/10.1073/pnas.1510502113.

29. Toh S, Rasmussen-Torvik $L$, Harmata EE, et al. The National PatientCentered Clinical Research Network (PCORnet) bariatric study cohort: rationale, methods, and baseline characteristics. JMIR Res Protoc. 2017;6(12):e222. https://doi.org/10.2196/resprot.8323.

30. Hripcsak G, Albers DJ. Next-generation phenotyping of electronic health records. J Am Med Inform Assoc. 2013;20(1):117-21. https://doi. org/10.1136/amiajnl-2012-001145.

31. Perotte A, Hripcsak G. Temporal properties of diagnosis code time series in aggregate. IEEE J Biomed Health Inform. 2013;17(2):477-83. https://doi. org/10.1109/JBHI.2013.2244610.

32. Elliott AF, Davidson A, Lum F, et al. Use of electronic health records and administrative data for public health surveillance of eye health 
and vision-related conditions in the United States. Am J Ophthalmol. 2012;154(6 Suppl):S63-70. https://doi.org/10.1016/j.ajo.2011.10.002.

33. Wilson J, Bock A. White Paper: The benefit of using both claims data and electronic medical record data in health care analysis. Eden Prairie, MN: Optum, Inc:; 2012. Available at: https://www.optum.com/content/dam/ optum/resources/whitePapers/Benefits-of-using-both-claims-and-EMRdata-in-HC-analysis-WhitePaper-ACS.pdf Accessed December 11, 2020.

34. Agniel D, Kohane IS, Weber GM. Biases in electronic health record data due to processes within the healthcare system: retrospective observational study. BMJ. 2018;361:k1479. https://doi.org/10.1136/bmj.k1479.

35. McPadden J, Durant TJ, Bunch DR, et al. Health care and precision medicine research: analysis of a scalable data science platform. J Med Internet Res. 2019;21(4):e13043. https://doi.org/10.2196/13043.

36. PCORnet. PCORnet Common Data Model (CDM). PCORnet Common Data Model (CDM) v3.1 2017. Available at: https://archive.pcornet.org/wpcontent/uploads/2016/11/2016-11-15-PCORnet-Common-Data-Model -v3.1_Specification.pdf Accessed December 11, 2020.

37. Blecker S, Katz SD, Horwitz LI, et al. Comparison of approaches for heart failure case identification from electronic health record data. JAMA Cardiol. 2016;1 (9):1014-20. https://doi.org/10.1001/jamacardio.2016.3236.

38. Hripcsak G, Shang N, Peissig PL, et al. Facilitating phenotype transfer using a common data model. J. Biomed. Inform. 2019:103253. https://doi. org/10.1016/j.jbi.2019.103253.

39. Weiskopf NG, Weng C. Methods and dimensions of electronic health record data quality assessment: enabling reuse for clinical research. J Am Med Inform Assoc. 2013;20(1):144-51. https://doi.org/10.1136/amiaj nl-2011-000681.
40. Weiner MG, Embi PJ. Toward reuse of clinical data for research and quality improvement: the end of the beginning? Ann Intern Med. 2009;151(5):359-60.

41. Hripcsak G, Albers DJ, Perotte A. Exploiting time in electronic health record correlations. J Am Med Inform Assoc. 2011;18(Suppl 1):1109-15. https://doi.org/10.1136/amiajnl-2011-000463.

42. Golladay KK, Collins AB, Ashcraft A, et al. Adverse Events in Hospitals: Methods for Identifying Events. Department of Health and Human Services; 2010:60.

43. Krishnamoorthy A, Peterson ED, Knight JD, et al. How Reliable are PatientReported Rehospitalizations? Implications for the Design of Future Practical Clinical Studies. J. Am. Heart Assoc. 2016;5(1). https://doi.org/10.1161/ JAHA.115.002695.

44. Electronic Medical Records and Genomics (emerge) Network. Available at: https://emerge.mc.vanderbilt.edu/about-emerge/. Accessed October 31, 2018.

45. Hripcsak G, Duke JD, Shah NH, et al. Observational health data sciences and informatics (OHDSI): opportunities for observational researchers. Stud Health Technol Inform. 2015;216:574-8.

\section{Publisher's Note}

Springer Nature remains neutral with regard to jurisdictional claims in published maps and institutional affiliations.
Ready to submit your research? Choose BMC and benefit from:

- fast, convenient online submission

- thorough peer review by experienced researchers in your field

- rapid publication on acceptance

- support for research data, including large and complex data types

- gold Open Access which fosters wider collaboration and increased citations

- maximum visibility for your research: over $100 \mathrm{M}$ website views per year

At BMC, research is always in progress.

Learn more biomedcentral.com/submissions 